ISSN: 1576-0162

\title{
Efectos macroeconómicos de las Cadenas Globales de Valor en la Balanza Comercial
}

\author{
Macroeconomic Effects of Global Value Chains \\ IN THE TRADE BALANCE \\ Pablo Ignacio Chena' \\ Universidad Nacional de La Plata. LESET-IdIHCS-CONICET \\ pablochena@gmail.com \\ Deborah Mercedes Noguera² \\ LESET-IdIHCS-CONICET \\ deborah_noguera@hotmail.com
} Recibido: marzo 2019; aceptado: octubre 2019

\section{RESUMEN}

El presente artículo analiza los efectos macroeconómicos de las Cadenas Clobales de Valor (CGV) sobre la balanza comercial, conjuntamente con la influencia de las mismas en las políticas económicas que tradicionalmente se diseñaron para modificar su resultado (por ejemplo: la devaluación real de la moneda doméstica). Con este objetivo se estima el impacto de las CGV en la balanza comercial en 48 países de América, Europa, Asia y Oceanía, durante el período 1995-2011. Los resultados alcanzados muestran que una mayor participación en las mismas genera un efecto directo de aumento del déficit de balanza comercial y, como efectos indirectos, un incremento en la elasticidad ingreso de las demandas por exportaciones e importaciones, conjuntamente con una disminución en la sensibilidad de los flujos comerciales a los cambios en el tipo de cambio real multilateral.

Palabras clave: Cadenas Globales de Valor; Balanza Comercial; Comercio Exterior; Datos de Panel.

\footnotetext{
' Investigador del Laboratorio de Estudios en Sociología y Economía del Trabajo- Instituto de Investigaciones en Humanidades y Ciencias Sociales - Consejo Nacional de Investigaciones Científicas y Técnicas - (LESET/IdIHCS/CONICET), Universidad Nacional de La Plata, La Plata, Argentina.

${ }^{2}$ Becaria doctoral con lugar de trabajo en el Laboratorio de Estudios en Sociología y Economía del Trabajo- Instituto de Investigaciones en Humanidades y Ciencias Sociales - Consejo Nacional de Investigaciones Científicas y Técnicas - (LESET/IdIHCS/CONICET), Universidad Nacional de La Plata, La Plata, Argentina.
} 


\section{ABSTRACT}

This article analyzes the macroeconomic effect of the Global Value Chains (CGV) on the trade balance, together with their influence on the economic policies that were traditionally designed to modify their result (for example: the real devaluation of the domestic currency). With this objective, we estimated their impact in the trade balance of 48 countries in America, Europe, Asia and Oceania, during the period 1995-2011. The results arrived show that a greater participation in them generates, as a direct effect, an increase in the trade balance deficit and, as indirect effect, an increase in the income elasticity of the demands for exports and imports, together with a decrease in the sensibility of trade flows to changes in the multilateral real exchange rate.

Keywords: Global Value Chains; Trade Balance; Foreign Trade; Data Panel.

Clasificación JEL / JEL classification: F14; F42; O50. 


\section{INTRODUCCIÓN ${ }^{3}$}

La teoría macroeconómica estándar atribuye a las decisiones económicas de cada país una autonomía suficiente para regular los flujos de comercio internacional en su territorio en función de objetivos internos, como el nivel de empleo, o externos como el equilibrio comercial. Esta concepción parte de estudiar los efectos de diferentes políticas macroeconómicas sobre la balanza comercial (como las devaluaciones reales de la moneda doméstica), bajo el supuesto de mercados internacionales donde los bienes producidos por cada paîs compiten entre sī.

Sin embargo, existe un grupo creciente de literatura que señala que la inserciōn internacional determina la tasa de crecimiento de la economía de un país y que la misma es relativamente exógena de las políticas macroeconómicas domésticas. Esta mirada recorre la teoría poskeynesiana de economía abierta (Thirlwall, 1979), el estructuralismo latinoamericano (Prebisch, 1968; Diamand, 1972), y el análisis histórico estructural del "sistema mundo" (Wallerstein, 1974), donde se destaca que la divisiōn internacional del trabajo está guiada por Cadenas Productivas integradas a escala global ${ }^{4}$, donde los Estados nacionales (especialmente periféricos y semiperiféricos) tienen poca influencia ${ }^{5}$.

La divergencia entre las mismas surge a la hora de explicar las causas de este fenómeno estructural. En el primer caso se debe a una rigidez del tipo de cambio real (McCombie, 1993), el segundo lo adjudica a asimetrías en las elasticidades ingreso de los bienes importados y exportados; 0 a divergencias en la organizaciōn de los mercados de trabajo (Prebisch, 1968); y el tercero a la

\footnotetext{
${ }^{3}$ Acknowledgements: We are grateful to two anonymous reviewers and to the editors of REM for valuable comments and suggestions. We are also in debt to Victor Troster and Ferran Portella-Carbó. Usual disclaimers apply. Funding: This work was supported by the research grant ¿Mediterranean Capitalism?: successes and failures of industrial development in Spain, 1720-2020, PGC2018 093896-B-100,directed by Jordi Catalan and financed by the Spanish Ministry of Economy; and by the research grant FPI/1866/2016, financed by the European Social Fund and the Ministry of Research, Innovation and Tourism of the Balearic Islands, which is gratefully acknowledged.

${ }^{4}$ Entendiendo las cadenas productivas como el conjunto de procesos productivos vinculados a un bien desde su concepciōn hasta el servicio que se realiza luego de su venta: "Entendemos por tales cadenas lo siguiente: tome un bien de consumo final y rastree el conjunto de insumos que culminó en este bien: las transformaciones anteriores, las materias primas, los mecanismos de transporte, la mano de obra en cada uno de los procesos materiales y los insumos de alimentos de dicha mano de obra. A este conjunto de procesos vinculados lo llamamos una cadena productiva." (Hopkins y Wallerstein, 1977).

${ }^{5}$ Esta descripciōn es simplemente enunciativa, a modo de ejemplo, y no pretende ser exhaustiva de las teorias que comparten esta mirada.
} 
forma de producción global del capitalismo (Wallerstein, 1974). En consonancia con esta última, estudios más recientes sobre Cadenas Globales de Valor (CGV) (Gereffi, 2014; Fernandez, 2014), alertan también sobre la capacidad limitada que tienen actualmente los países para modificar, a través de las políticas macroeconómicas, los flujos de comercio internacional y la divisiōn internacional del trabajo generada en las decisiones productivas de las empresas líderes que gobiernan dichas Cadenas a escala mundial (Humphrey y Schmitz, 2002).

La presente investigación tiene como motivaciōn principal profundizar el análisis de los cambios estructurales que la inserción en CGV produce en los flujos comerciales de los países, en funciōn de sus niveles de desarrollo económico relativo. Así como también las modificaciones en los márgenes de maniobra que las CGV imponen a las políticas macroeconómicas internas diseñadas para alterarlos. En este marco, el artículo tiene dos objetivos específicos. El primero es dimensionar el impacto económico directo de una mayor participaciōn en CGV sobre los volúmenes de importaciones y exportaciones globales, tanto en países de ingresos altos (desarrollados) como de ingresos medios (en desarrollo). El segundo, estimar los efectos indirectos que dicho fenómeno genera en las elasticidades precios e ingreso del comercio exterior de los países involucrados. Para esto se analiza econométricamente un panel con datos de 48 paîses, clasificados según su nivel de ingreso.

El artículo se estructura de la siguiente manera, en la sección 2 se revisa la literatura teórica y empírica referida al efecto de las CGV en el comercio internacional; en la sección 3 se estima econométricamente la influencia de las mismas en 48 países de América, Europa, Asia y Oceanía durante el período 1995-2011 y, finalmente, en la secciōn 4 se esbozan las reflexiones finales.

\section{Las Cadenas Globales de Valor y su impacto macroeconómico}

La literatura especializada advierte que las CGV pueden afectar la efectividad de las políticas macroeconómicas (Smichowski et al. 2016); ya que estān dominadas por firmas con capacidad para "influir en las actividades de otras empresas de la cadena (...). Este poder es ejercido mediante el control a la entrada y salida de empresas en la cadena, monitoreo a los proveedores y el control de las empresas lideres en recursos claves" (Gereffi et al., 2001, p. 5). Con el objetivo de analizar la gobernanza al interior de las CGV, (Gereffi et al., 2005) propone una tipología con cinco posibilidades: de mercado, modular, relacional, cautiva y jerárquica.

En la gobernanza de mercado la relaciōn entre las empresas es horizontal y funciona cuando la complejidad del intercambio de informaciōn entre proveedor y comprador es baja y altamente codificable. En el otro extremo, la jerárquica suele operar cuando la información que se transfiere entre compradores y proveedores es compleja y difícilmente codificable. En este último caso, la firma líder de la cadena internaliza las actividades y los vínculos entre filiales de una misma empresa, por lo que se requieren elevados grados de coordina- 
ciōn. En la gobernanza modular y relacional, existen interacciones complejas entre compradores y vendedores, que a menudo crean dependencia mutua, y la confianza entre las partes se vuelve importante. Finalmente, bajo un tipo de gobernanza cautivo, los proveedores dependen transaccionalmente de compradores mucho más grandes y se enfrentan a costos significativos si desean cambiar de socio; lo que los vuelve "cautivos" de un alto grado de supervisión y control por parte de las empresas líderes.

De lo anterior se deduce que las medidas macroeconómicas tradicionales, que basan su eficacia en el funcionamiento adecuado del sistema de precios relativos, tienen un efecto deseado solo en la forma de gobernanza de mercado. Para el resto de las posibilidades, el sistema de gobierno de la CGV se vuelve un límite al efecto de dichas políticas en la asignación de recursos.

En el caso de los países en desarrollo, a lo anterior se agrega un cuerpo creciente de literatura que muestra que los esquemas de gobernanza de las CGV que dominan en dichas regiones son del tipo cautivos (Bair y Gereffi, 1998); debido a factores como:

i) La brecha de capacidad productiva que existe entre los proveedores locales y la que exigen las industrias de exportación (Humphrey y Schmitz, 2002); lo que obliga a los compradores globales a invertir en ciertos proveedores para alcanzar los estándares de calidad requeridos por la competencia.

ii) Una elevada concentración económica en el sector minorista de la cadena que, combinado con un número creciente de proveedores intensivos en mano de obra procedentes de los países en desarrollo, genera un poder de negociación asimétrico a favor de los compradores globales.

iii) La necesidad de innovaciones específicas para algunos consumidores que, junto con una tendencia creciente a reducir los costos de inventario, incentivan relaciones cautivas para evitar fallas productivas en las CGV.

Finalmente, estos sistemas de gobernanza tipo cautivo que priman en los países en desarrollo llevan a algunos autores a destacar que la integración en CGV puede ser una espada de doble filo para los mismos porque, por un lado, "las empresas de los países en desarrollo pueden exportar a mercados que de otro modo les sería dificil penetrar" y, por otro, "se vinculan a relaciones que evitan la actualización funcional y los dejan dependientes de un pequeño número de clientes poderosos. En algunos casos, las relaciones exclusivas con grandes compradores les impiden diversificar su base de clientes. Esto aumenta aún más el costo de la 'opción de salida', atándolos a su comprador clave" (Humphrey y Schmitz, 2002: 1024).

En este sentido, la relación entre las características de la especializaciōn productiva de cada país y la estructura de gobierno de las CGV, resulta de especial relevancia a la hora de evaluar los efectos de incrementar su participación en las mismas. En particular, destacamos sus consecuencias sobre el crecimiento económico y sobre la efectividad de ciertas políticas macroeconómicas diseñadas a escala nacional para influenciar los flujos de comercio internacional en base a objetivos de desarrollo interno. 


\subsection{LOS EFECTOS SOBRE EL COMERCIO EXTERIOR}

Existe cierto consenso en la literatura de que un aumento del comercio mundial a través de CGV modifica en forma directa los saldos comerciales y de cuenta corriente de los países involucrados (Banco Mundial, 2017). Sin embargo, la evidencia empírica sobre el sentido de los mismos es nuevamente controversial. Por un lado, Georgiadis et al. (2014) y Brumm et al. (2019) sugieren, por ejemplo, que la participación en CGV debería tener un efecto positivo en la balanza comercial de los países porque el proceso implica agregar valor a las importaciones y luego exportarlas, lo que mejora la balanza comercial. En esta línea, mediante un análisis de panel con datos de 29 países en el período 1995-2011, el ECB Economic Bulletin (2017) encuentra que incrementar la participación en CGV tiene un impacto positivo en el saldo de su cuenta corriente y que, por lo tanto, las diferencias de participación en CGV explican una parte sustancial de los superávits de cuenta corriente que se observaron en el período previo a la crisis financiera de 2008.

Por otra parte, Haltmaier (2015) argumenta que los estudios que encuentran efectos positivos de la integración en CGV sobre la cuenta corriente no incorporan al análisis la posición que ocupan los países en las mismas. Mediante un análisis de panel para 40 países el autor encuentra que una mayor participación en CGV tiene un efecto global negativo en el saldo comercial de los mismos y que éste es menor en el caso de aquellos que se posicionan en eslabonamientos hacia adelante en la cadena de producción (upstream). Por su parte, López-Villavicencio y Mignon (2018) Ilegan a conclusiones similares, con la evidencia de un panel de 57 países centrales y periféricos, donde muestran que incrementar la participaciōn escalando hacia eslabonamientos aguas abajo tiene un efecto negativo sobre el saldo de la balanza comercial.

A esto se suman importantes efectos "indirectos" de las mismas sobre la capacidad de regulación de los flujos comerciales a nivel nacional. Consecuencias que se reflejan en las elasticidades ingreso y precios de las exportaciones e importaciones como se observa a continuaciōn.

\subsubsection{ANÁLISIS DE LAS ELASTICIDADES INGRESO DE EXPORTACIONES E IMPORTACIONES}

A nivel global, un cúmulo creciente de estudios destaca, como hecho estilizado, un incremento en la sensibilidad de los flujos de comercio internacional de cada país respecto del ingreso (doméstico y de los socios comerciales) para los últimos 30 años (Irwin, 2002; Freund, 2009; Escaith, 2009; Constantinescu et al., 2015). Se observa también que las CGV han contribuido a este fenómeno incrementando el grado de interconexiōn que producen entre las economías nacionales. Por ejemplo, Escaith et al. (2010) encuentran que la expansiōn de las CGV determinó un aumento de la elasticidad ingreso del comercio exterior a nivel nacional, especialmente a partir de la década de 1980. Mientras que para Bems et al. (2010), la fragmentación de la producción mun- 
dial puede amplificar el impacto de los shocks de demanda internacional y justificar asî elasticidades-ingreso mayores que uno. Por otra parte, Bussiere et al. (2013) concluyen, con la evidencia de un panel de 18 países, que la fragmentaciōn de la producciōn mundial contribuyó a profundizar la caída del comercio internacional en la crisis económica de 2008-2009, al incrementar las elasticidades ingreso de las importaciones. Este fenómeno se debería a que la integraciōn en CGV implica un alto contenido de importación en las exportaciones y, a su vez, una fuerte sincronizaciōn de las transacciones comerciales entre los países.

Por otra parte, Buono y Vergara Caffarelli (2013) destacan, utilizando datos de panel de 38 países para el período 1998-2009, que la especialización vertical puede aumentar las elasticidades de las exportaciones e importaciones respecto al ingreso y que los grandes shocks de demanda doméstica inducen a las empresas a variar el grado de especialización vertical ${ }^{6}$. Los autores sostienen que una caída en la demanda final destruye flujos comerciales y reasigna internamente etapas de la producción que se encontraban externalizadas. A la inversa, durante los períodos de auge, el establecimiento de nuevas redes de especializacioon vertical crea flujos comerciales y, en consecuencia, un aumento más que proporcional del comercio mundial. De esta forma, los cambios en la especializaciōn vertical, producto de variaciones de demanda, afectan la elasticidad ingreso de las exportaciones e importaciones.

Por último, Constantinescu et al. (2015) analizan la relación entre comercio internacional e ingreso nacional, durante el período 1970-2013, y encuentran que la elasticidad-ingreso de las importaciones a largo plazo aumentó marcadamente en la década de los noventas y bajó en la década posterior. Al analizar los determinantes de esta última retracción observan que un ritmo más lento en la expansiōn de las CGV es un factor causal significativo de este fenómeno. Finalmente, sostienen que la elevada elasticidad ingreso de largo plazo de las importaciones observada en los años 90 refleja la creciente fragmentaciōn de la producciōn impulsada principalmente por Estados Unidos y China; y que ese impulso particular se agotó en la década posterior.

Sin embargo, en contraste con los hallazgos anteriores, Bénassy-Quéré et al. (2009) computando un modelo de equilibrio general multisectorial y multipaîs que incorpora las relaciones interindustriales dentro y entre paîses, concluyen que la fragmentación de las cadenas globales es consistente con una reacción proporcional del comercio al PBI mundial. Es decir, que las mismas no contribuyen a incrementar la sensibilidad de los flujos comerciales ante cambios en el producto.

\footnotetext{
${ }^{6}$ Un cambio en el grado de especializaciōn vertical ocurre en el caso de una renacionalizaciōn de una etapa de producción que se encontraba en el exterior o, a la inversa, con la internacionalización de una etapa de la producción que se realizaba localmente.
} 


\subsubsection{SOBRE LAS ELASTICIDADES PRECIO DE LAS EXPORTACIONES E IMPORTACIONES}

En los últimos años diversos trabajos dan cuenta de la reducción de la sensibilidad de los flujos comerciales ante variaciones del tipo de cambio real, especialmente en el caso de las exportaciones (Eichengreen y Gupta, 2013; Ahmed et al. , 2015; Chena y Bosnic, 2017). En esta línea, Ahmed et al. (2015) muestra que la concentración de exportaciones en las CGV disminuye la sensibilidad de las mismas ante cambios en el tipo de cambio real, debido a los encadenamientos hacia atrás y hacia adelante que genera entre producciones de una misma cadena ubicadas en diferentes regiones. Por otra parte, Amiti et al. (2014) destacan que, para las empresas con una alta participación de mercado y un elevado número de componentes importados en la producción, el impacto del tipo de cambio en el precio de exportación es bajo; mientras que, en el caso de los pequeños exportadores con una baja proporción de componentes importados, este efecto es máximo. Los autores señalan que dicho comportamiento se debe al impacto de las importaciones sobre el costo marginal y al efecto del poder de mercado sobre la determinación de los precios. Por lo tanto, cuando las exportaciones son más intensivas en componentes importados y la participaciōn de mercado de las firmas exportadoras en el mercado mundial es mayor, el efecto del tipo de cambio real en los flujos comerciales es menor (Amiti et al., 2014).

En línea con estos resultados, Bayoumi et al. (2013) encuentran que, debido a la distribución de la producción a través de las CGV, los cambios en los precios relativos de los bienes se han vuelto menos sensibles a los movimientos de los precios internos de los factores. Es decir que la participación en CGV aumentó la rigidez del tipo de cambio real respecto a factores domésticos, especialmente para las economías periféricas. En este contexto, una pérdida de competitividad debido a un aumento en los costos relativos de los factores no se traduce necesariamente en una pérdida de competitividad de los bienes. Por su parte, Kharroubi (2011) muestra que el mayor grado de especialización vertical, producto del crecimiento de las cadenas de suministro globales, actúan reduciendo la sensibilidad de los flujos comerciales a cambios en los precios relativos. Por lo tanto, el ajuste del tipo de cambio real no es una solución en sí misma para corregir desbalances de cuenta corriente, sino que debe ir acompañado de medidas adicionales.

Con el propósito de proporcionar nueva evidencia a este debate, en la siguiente secciōn se estiman las elasticidades del comercio exterior para un panel con 48 países de distintas regiones del mundo entre finales del siglo XX y principios del siglo XXI. En el modelo se incluyen como variables la participación en CGV y su interacción con el tipo de cambio real y el ingreso (doméstico y externo), con el objetivo de evaluar los efectos directos e indirectos de este fenómeno en la balanza comercial. 


\section{Estimación empírica de los efectos de LAs CGV SOBRE El COMERCIO INTERNACIONAL}

Para estimar la relevancia de la integraciōn en CGV sobre los flujos de comercio de los 48 países seleccionados (Tabla A1 Anexo) se partió del modelo reducido de balanza comercial de Goldstein y Khan (1985), donde la misma depende negativamente del ingreso doméstico y del tipo de cambio real multilateral ${ }^{7}$, y positivamente del ingreso foráneo.

La incorporación de los efectos directos e indirectos de una mayor parti-

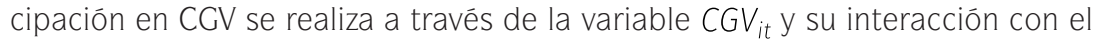
tipo de cambio real y el producto (doméstico y externo), como se expresa en el modelo $(1)^{8}$.

$B C_{i t}=\beta^{*} Y_{i t}^{*}+\beta Y_{i t}+\eta T C R M_{i t}+\delta C G V_{i t}+\gamma C G V x T C R M_{i t}+\rho C G V x Y_{i t}+\omega C G V x Y_{i t}^{*}+u_{i t}$

Donde $B C_{i 1}$ es la balanza comercial, $T C R M_{i t}$ es el tipo de cambio real multilateral (doblemente ponderado); $Y_{i t}^{*}$ es el ingreso mundial; e $Y_{i t}$ es el ingreso doméstico; y las elasticidades precio e ingreso del comercio exterior están expresadas respectivamente por $\eta$ y $\beta, \beta^{*}$.

La variable $C G V_{i t}$ indica el grado de participaciōn en CGV para el país i y nos permite ver el efecto directo sobre la balanza comercial. Luego se incluyen tres términos de interacción de esta variable con: a) el tipo de cambio real (CGVxTCRM $M_{i t}$; b) el ingreso doméstico ( $\left.C G V x Y_{i t}\right)$ y c) el ingreso extranjero $\left(C G V X Y_{i t}^{*}\right)$. Lo que permite evaluar el impacto de la integración en CGV sobre la sensibilidad de la balanza comercial ante cambios en cada una de esas variables.

El efecto teórico directo que tiene aumentar la participación en CGV sobre la balanza comercial difiere según la posiciōn que los países ocupen en la misma (Haltmaier, 2015). Si el proceso de integraciōn implica agregar valor a insumos o materias primas importadas y luego exportar el producto final, se espera que un aumento en la integraciōn en CGV tenga un efecto positivo sobre la balanza comercial (Georgiadis et al., 2014). Por el contrario, si el país se integra especialmente como exportador de materias primas o productos intermedios para su posterior procesamiento en otros lugares el efecto será negativo. Por lo tanto, el signo del coeficiente que acompaña a CGV dependerá del efecto que predomine en cada caso.

En cuanto a la interacciōn entre $C G V_{i t}$ y $T C R M_{i t}$, la literatura revisada en la secciōn previa indica que tendrá un coeficiente con signo positivo (contario al efecto directo del TCRM sobre la Balanza Comercial), para destacar que

\footnotetext{
${ }^{7}$ Por construcciōn, un incremento en el tipo de cambio real (TCR) es equivalente a una apreciaciōn de la moneda doméstica. Se espera entonces que una devaluaciōn del TCR tenga efectos positivos en la balanza comercial.

${ }^{8}$ Las variables se expresan en logaritmos.
} 
una mayor integración en CGV reduce la reacciōn de los flujos comerciales al TCRM.

Respecto al ingreso, se espera un signo positivo en la interacción de CGV $_{\text {it }}$ con el ingreso mundial y negativo con el doméstico, reforzando los efectos directos; debido a que el grado de interconexión entre las economías aumenta a medida que se incorporan en CGV, dando lugar a una mayor sensibilidad del comercio ante cambios en los ingresos. Tal como argumentan Escaith et al. (2010), con el crecimiento de las redes de suministro los insumos intermedios cruzan la frontera en más de una oportunidad antes de que el producto terminado sea entregado al consumidor final. En este sentido, las diferentes etapas de producción de las CGV dependen unas de otras, como proveedores y como clientes. Por lo tanto, si se produce un shock ${ }^{9}$ en uno de los sectores o países participantes, el mismo se transmite rápidamente a las otras etapas de la CGV a través de los encadenamientos hacia atrás y hacia adelante.

Finalmente, para analizar la robustez de los resultados se evalúan también los efectos de la participación en CGV sobre la sensibilidad de la balanza comercial al tipo de cambio real a través de la variable $I C E_{i t}$, que expresa el contenido importado en las exportaciones como porcentaje de las importaciones totales. En otras palabras, representa el grado en que un país se encuentra especializado verticalmente en el comercio internacional, ya que mide la contribución de las importaciones en la producción de bienes y servicios exportados.

Al incorporar este efecto el modelo queda definido de la siguiente manera:

$$
B C_{i t}=\beta^{*} Y_{i t}^{*}+\beta Y_{i t}+\eta T C R M_{i t}+\varphi I C E_{i t}+T \text { ICEXTCRM } M_{i t}+v_{i t}
$$

Cuanto más especializado verticalmente se encuentra un país, mayor es la dependencia que el volumen de exportaciones tiene del volumen de importaciones necesarias para la producciōn $I C E_{i t}$. Por ello, se espera que el signo de $\varphi$ sea negativo; ya que un incremento en el contenido de importaciones en las exportaciones de un país afecta negativamente al saldo de la balanza comercial. Respecto al signo del coeficiente $T$, se espera que sea positivo porque una mayor dependencia de insumos importados en las industrias de exportaciōn, o bien un co-movimiento más estricto entre exportaciones e importaciones, debería reducir la sensibilidad de la balanza comercial al tipo de cambio real.

\footnotetext{
${ }^{9}$ Por ejemplo, una crisis crediticia en un país integrado, un cambio en la política comercial, un aumento de aranceles, la imposiciōn de barreras no arancelarias y la implementación de campañas de "compra local", entre otras cosas.
} 


\subsection{LOS DATOS Y LA METODOLOGÍA DE ESTIMACIŌN}

La estimación se realiza con datos anuales de producto bruto interno (PBI), exportaciones e importaciones en valores reales, tipo de cambio real multilateral y contenido de valor agregado en el comercio internacional para un panel de 48 países durante el período 1995-2011 (Tabla A1 del anexo) ${ }^{10}$. La construcción de cada variable y la fuente de los datos utilizada se detallan en la tabla 1.

Para el análisis econométrico se utiliza la metodología de datos en panel. El resultado del test de Hausman (tabla A2) indica que los efectos individuales están correlacionados con las variables explicativas; por lo tanto, se opta por la utilización del modelo de efectos fijos. En particular, se incorporan efectos fijos de corte transversal, para controlar por las diferencias propias de cada país, y temporales, para tomar en cuenta aquellos factores específicos que con el tiempo puedan influir en los resultados.

La especificación completa de los modelos de regresión es la siguiente:

$$
\begin{array}{r}
B C_{i t}=\beta^{*} Y_{i t}^{*}+\beta Y_{i t}+\eta T C R M_{i t}+\delta \operatorname{CGV}_{i t}+Y \operatorname{CGVXTCRM}_{i t}+\mu_{i}+\mu_{t}+\varepsilon_{i t} \text { (3) } \\
B C_{i t}=\beta^{*} Y_{i t}^{*}+\beta Y_{i t}+\eta T C R M_{i t}+\delta C^{*} V_{i t}+\rho \operatorname{CGVXY}_{i t}+\omega \operatorname{CGVXY} Y_{i t}^{*}+\mu_{i}+\mu_{t}+\varepsilon_{i t}
\end{array}
$$

Donde $\mu_{i}$ y $\mu_{t}$ son los efectos fijos específicos de sección transversal y de tiempo respectivamente. Con la estimación del modelo (3) se obtienen los resultados de la integración en CGV sobre la sensibilidad de los flujos comerciales al TCRM. Mientras que el modelo (4) explicita la sensibilidad de los flujos comerciales ante variaciones en el producto bruto (doméstico y foráneo).

A esto se suman estimaciones para dos sub-muestras correspondientes a países de ingreso alto (desarrollados) y de ingreso medio (en desarrollo) -clasificados según el criterio del Banco Mundial'1"-, con el objetivo de evaluar la robustez de los resultados (ver anexo).

\subsection{Resultados}

Los resultados alcanzados se resumen en la tabla 2. Las primeras columnas ((i) a (iii)) muestran el efecto directo de la integraciōn en CGV sobre la balanza comercial, mientras en las siguientes tres ((iv) a (vi)) destacan los efectos de la participación en CGV sobre la sensibilidad de los flujos comerciales al tipo de cambio real. Las últimas tres columnas ((vii) a (ix)) explicitan el efecto de la integración en CGV sobre las elasticidades ingreso.

\footnotetext{
${ }^{10}$ El período seleccionado está vinculado a la disponibilidad de datos para la variable "indicador de participaciōn en CGV", fuente TiVA- OCDE (diciembre 2016).

${ }^{11}$ Para mayor detalle sobre la clasificaciōn de los países que realiza el Banco Mundial ver: https:// datahelpdesk.worldbank.org/knowledgebase/topics/19280-country-classification.
} 


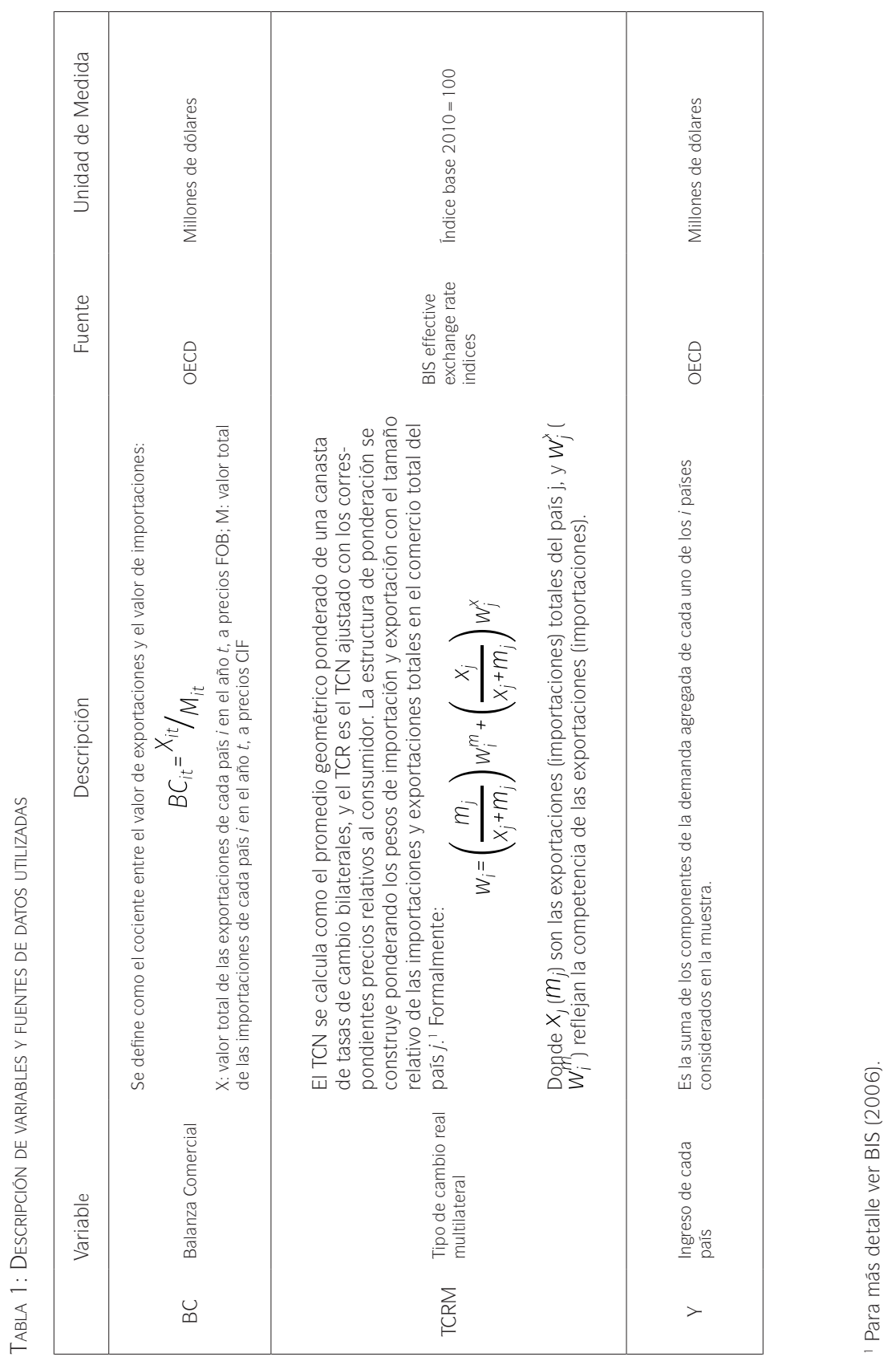




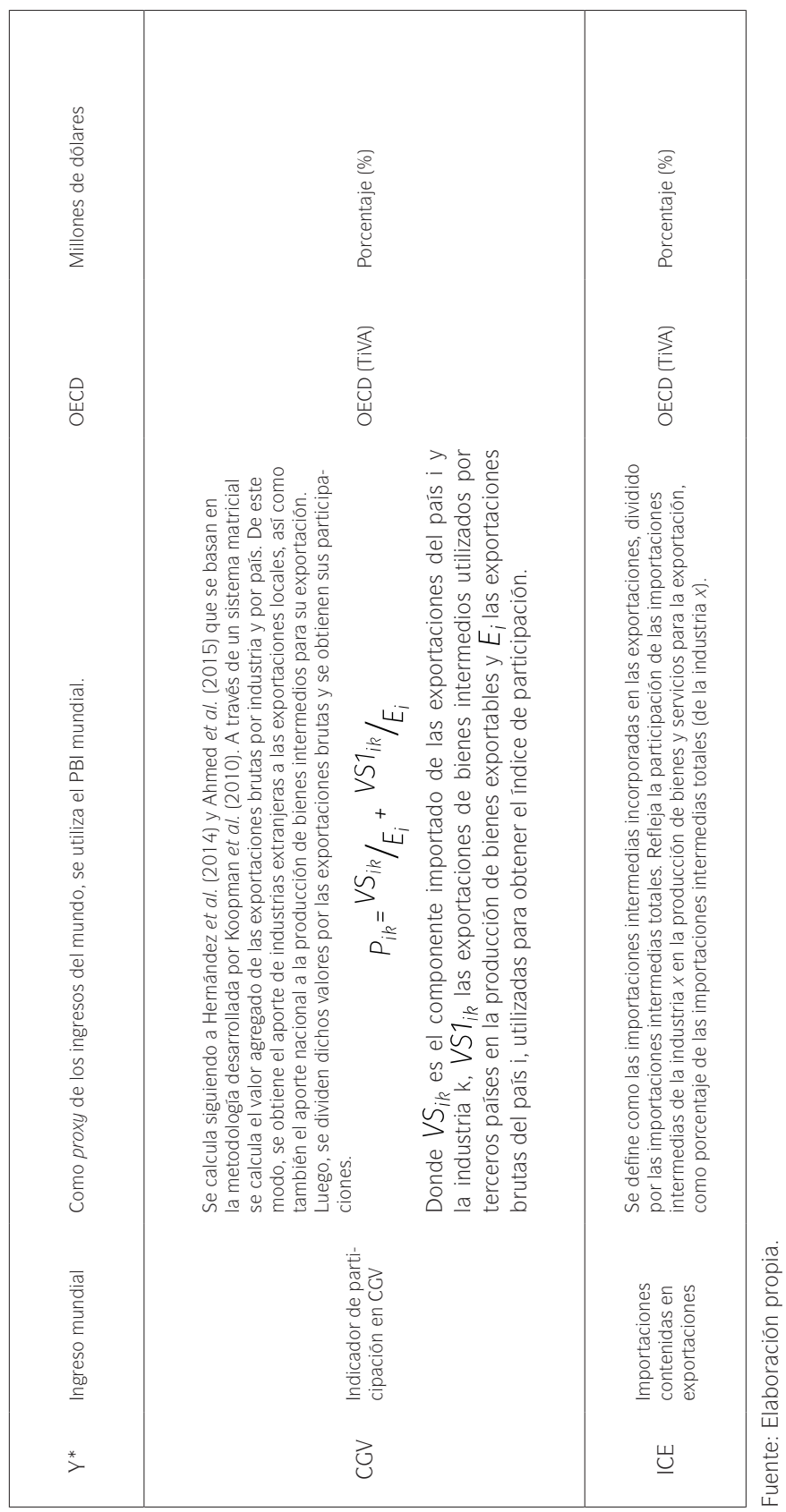




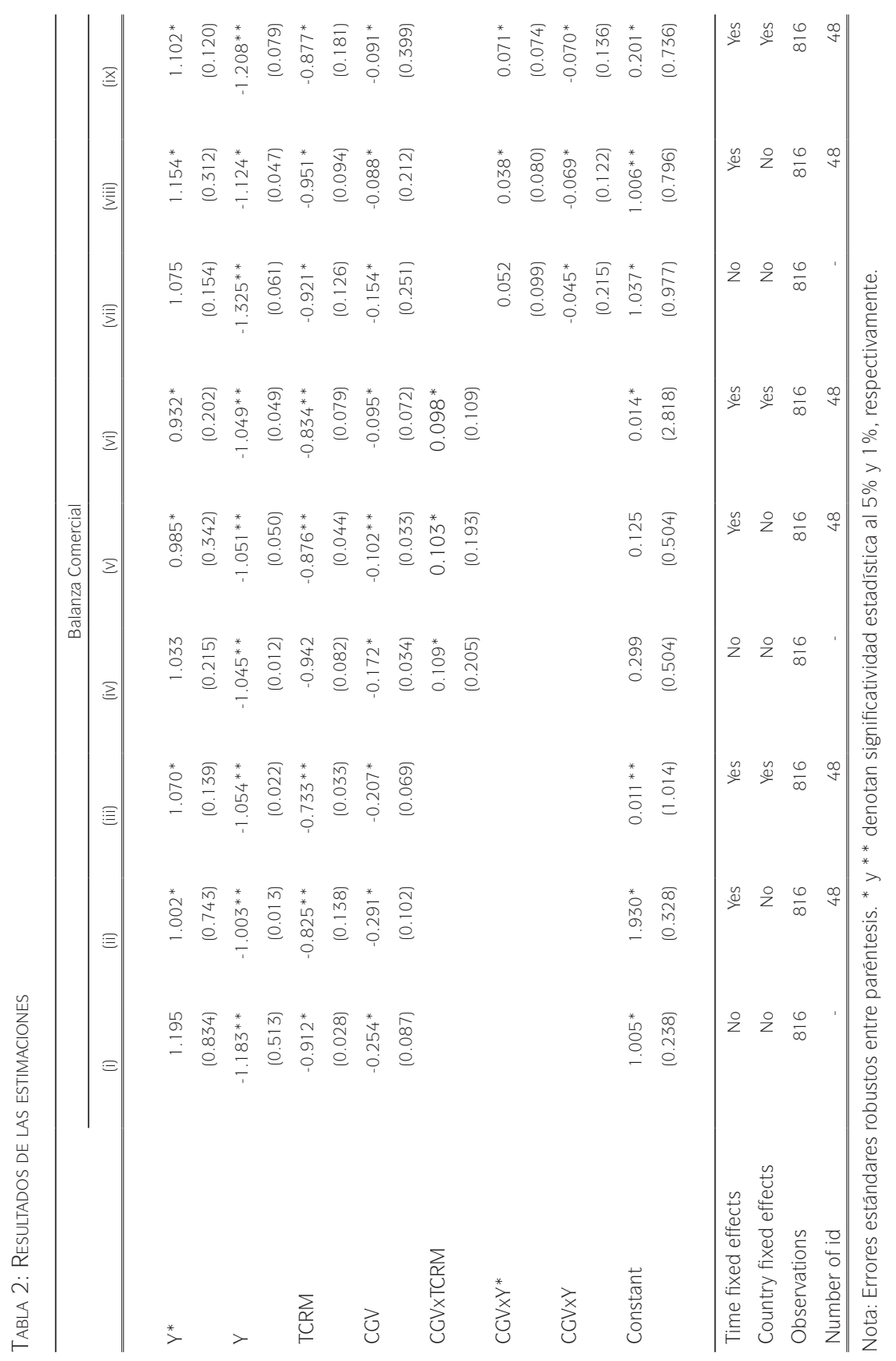


A lo largo de todas las estimaciones, los coeficientes de las variables $Y_{t}$ e $Y_{t}^{*}$ (ingreso del país i e ingreso mundial, respectivamente), tienen el signo esperado por la teoría económica y resultan estadísticamente significativos ${ }^{12}$. Esto indica que cuando un país crece, manteniéndose sin cambios el resto de las variables, experimenta una reducción en su superávit comercial (o un incremento de su déficit) y viceversa.

El TCRM resulta negativo y estadísticamente significativo en todas las estimaciones realizadas ${ }^{13}$. Lo que hace prever que, manteniendo fijas las demás variables, una apreciación del TCRM impacta negativamente en el saldo de la balanza comercial, mientras que una depreciaciōn tiene el efecto contrario.

El efecto directo que tiene la integración en CGV sobre la balanza comercial resulta negativo y estadísticamente significativo a un $5 \%$ en todas las estimaciones realizadas. Por lo tanto, un incremento marginal en la participaciōn en CGV desmejora el saldo de la balanza comercial al disminuir las exportaciones netas.

Respecto del coeficiente de interacción entre CGV $_{\text {it }}$ y TCRM, el mismo es positivo y estadísticamente significativo a lo largo de todas las estimaciones ensayadas. Este resultado sostiene la hipótesis de que la integración en CCV reduce la sensibilidad de los flujos comerciales ante variaciones en el TCRM. De acuerdo con las estimaciones realizadas, una depreciación de un $1 \%$ en el TCRM mejora la balanza comercial en un 0,54\% para un país en el cuartil inferior de la distribución del índice de participaciōn en CGV; mientras que esta cifra disminuye a 0,31\% para un país en el cuartil superior de la distribución del indice de participaciōn en CGV ${ }^{14}$.

Finalmente, los términos de interacciōn de la variable $C G V_{i t}$ con el producto doméstico y de los socios comerciales resultan estadísticamente significativos cuando se controla por efectos fijos individuales y temporales. En ambos casos, la interacción tiene el mismo signo que el efecto directo de cada variable sobre la balanza comercial. Esto indica que la misma incrementa la sensibilidad de los flujos comerciales respecto de $Y$ e $Y^{*}$.

Es importante señalar que los resultados alcanzados resultan robustos también cuando se mide el efecto de las CGV sobre los flujos comerciales y su sensibilidad ante cambios en el tipo de cambio real a travês de la variable ICE, que representa a las importaciones contenidas en los productos exportados como proporciōn de las importaciones totales (ver Tabla A3 del Anexo). En este sentido, la integración en CGV (medida mediante ICE) tienen un efecto directo negativo y significativo sobre la balanza comercial y el término de interacciōn (ICEXTCRM) resulta negativo y estadísticamente significativo al controlar por

\footnotetext{
${ }^{12}$ Excepto, para el caso del ingreso mundial, cuando el modelo se corre ignorando la existencia de heterogeneidad a nivel paîs y efectos fijos en el tiempo, ya que resulta no significativo (ver columnas (i) y (iv) de la Tabla 3).

13 A excepción del caso del modelo sin efectos fijos, en el cual se incluye la interacción de CGV con el tipo de cambio real.

${ }^{14}$ Los efectos marginales del TCRM sobre la balanza comercial están dados por: $\frac{\partial B C}{\partial T C R M}=\eta+\gamma$ CGV
} 
efectos fijos. Esto último, muestra que ante un aumento en el contenido importado de las exportaciones, producto de una mayor participación en CGV, los flujos comerciales serán menos sensibles a variaciones cambiarias reales.

Dichos resultados se mantienen, en términos de signo y significatividad de los coeficientes, en las estimaciones de las dos submuestras, donde se agrupa a los países según nivel de ingreso siguiendo el criterio del Banco Mundial (ver tabla A4 del Anexo). En ambos grupos de países una mayor participación en CGV tiene un efecto directo negativo y estadísticamente significativo sobre la balanza comercial, siendo el tamaño del efecto levemente mayor en los países de ingreso medio. En línea con este resultado, el término de interacciōn entre la participación en CGV y el TCRM (CGVXTCRM) indica que una mayor participación en CGV se asocia a una menor sensibilidad de los flujos comerciales ante variaciones cambiarias reales y este efecto es mayor, en términos absolutos, en los países de ingreso medio.

\section{REFLEXIONES FINALES}

El análisis de los resultados alcanzados en esta investigaciōn, bajo el prisma de las políticas macroeconómicas para el desarrollo, permite arribar a dos conclusiones relevantes. Por un lado, los hallazgos empíricos convalidan la hipótesis de que incrementar la participación en CGV refuerza la restricción externa en aquellos países cuyo crecimiento se encuentra restringido por la balanza de pagos (Thirlwall 1979). En este sentido, se observa que una mayor integración en las mismas tuvo consecuencias negativas directas sobre una muestra de 48 países de América, Europa, Asia y Oceanía. Como asī también sobre las submuestras de países de ingresos altos y medios; con un mayor efecto sobre los últimos.

Por otro lado, las estimaciones destacan que la tendencia global del capitalismo a estructurarse en CGV limita la eficacia de las políticas de tipo de cambio real elevado como instrumento central para promover un crecimiento liderado por las exportaciones netas (Hausmann et al., 2005; Frenkel y Ros, 2006). En este sentido, Ios resultados fortalecen la evidencia empírica referida a que una participación elevada en CGV reduce los efectos del TCRM sobre la balanza comercial y potencia la sensibilidad de los flujos comerciales a los ingresos domésticos y externos.

A esto se suma que para los paîses de ingresos medios (dentro de los cuales se encuentran los seleccionados para América Latina), los flujos comerciales son menos sensibles a las modificaciones en el TCRM que en aquellos de ingresos altos. Al tiempo que un incremento en el grado de participación en CGV reduce dicha sensibilidad en una mayor magnitud. Esto último impone fronteras estrictas a las posibilidades que tienen dichos países para desarrollarse sin una estrategia previa que controle la forma de inserción en las mismas. 


\section{REFERENCIAS}

Ahmed S., Appendino M. y Ruta M. (2015): "Depreciations without Exports? Global Value Chains and the Exchange Rate Elasticity of Exports", World Bank Policy Research Working Paper, 7390, disponible en: http://documents.worldbank.org/curated/en/689841468189545684/pdf/WPS7390. pdf.

Amiti M., Itskhoki O. y Konings J. (2014): "Importers, Exporters, and Exchange Rate Disconnect," The American Economic Review, 7(104), pp. 1942-78, disponible en: http://dx.doi.org/10.1257/aer.104.7.1942.

Bair J. y Gereffi G. (1998): "Interfirm Networks and Regional Divisions of Labor: Employment and Upgrading in the Apparel Commodity Chain." En: Conference on Global Production and Local Jobs, Geneva: International Labor Organization (ILO).

Banco Mundial; IDE-JETRO; OCDE; UIBE y OMC (2017): "Global Value Chain Development Report 2017: Measuring and Analyzing the Impact of GVCs on Economic Development", Washington, DC: World Bank. https://openknowledge.worldbank.org/handle/10986/29593.

Bayoumi, T.; Saito, M. y Turunen, J. (2013): "Measuring Competitiveness: Trade in Goods or Tasks?" Working Paper, International Monetary Found.

Bems, R.; Johnson, R. y Yi, K. (2010): "Demand Spillovers and the Collapse of Trade in the Global Recession," Working Paper, International Monetary Found.

Bénassy-Quéré, A; Decreux, Y.; Fontagné, L. y Khoudour-Casteras D. (2009): "Economic Crisis and Global Supply Chains", Centre d'études prospectives et d'informations internacionales, Working Paper $n^{\circ}$ 2009-15.

BIS (2006): "The New BIS Effective Exchange Rate Îndices”, BIS Quarterly Review.

Brumm, J., Georgiadis, G., Gräb, J., y Trottner, F. (2019): "Global Value Chain Participation and Current Account Imbalances", Journal of International Money and Finance.

Bussiére, M.; Callegari, F.; Sestieri, G. y Yamano, N. (2013): "Estimating Trade Elasticities: Demand Composition and the Trade Collapse of 2008-2009," American Economic Journal: Macroeconomics, 5(3), pp, 118-151, disponible en: http://dx.doi.org/10.1257/mac.5.3.118.

Buono, I. y Vergara Caffarelli, F. (2013): "Trade Elasticity and Vertical Specialization, Banca d'Italia Eurosistema, temi de discossiones”, Working Paper $n^{\circ} 924$.

Carneiro, F. (2015): "Fragmentação internacional da produção e cadeias globais de valor", en IPEA, 2097.

Chena P. y Bosnic C. (2017): "Concentración económica y comercio internacional. La condiciōn Marshall-Lerner en la Argentina (1993-2013)”, Cuadernos de Economía, 36(71), pp. 379-403.

Constantinescu, C.; Matoo, A. y Ruta, M. (2015): "The Global Trade Slowdown: Cyclical or Structural?”, Working Paper, International Monetary Found. 
Diamand, M. (1972): "La Estructura Productiva Desequilibrada y el Tipo de Cambio”, Desarrollo Económico, 12 (45), pp. 25-47.

ECB Economic Bulletin (2017): "The Impact of Global Value Chain Participation on Current Account Balances - a Global Perspective", European Central Bank Economic Bulletin, issue 2/ 2017, article 1.

Eichengreen, B. y Gupta, P. (2013): "The Real Exchange Rate and Export Growth: Are Services Different?” Policy Research Working Paper Series 6629, The World Bank.

Escaith, H. (2009): "Trade Collapse, Trade Relapse and Global Production Networks: Supply Chains in the Great Recession”, WTO Staff Working Papers.

Escaith, H.; Lindenberg, N. y Mirodout, S. (2010): "International Supply Chains and Trade Elasticity in Times of Global Crisis”, Working Paper, World Trade Organization, Economic Research and Statistics Division.

Fernández, R. (2014): "Global Value Chains in Global Political Networks: Tool for Development or Neoliberal Device?” Review of Radical Political Economics.

Frenkel, R. y Ros J., (2006); "Unemployment and the Real Exchange Rate in Latin America", en World Development, vol. 34 (4), pp. 631-646.

Freund, C. (2009): "The Trade Response to Global Downturns: Historical Evidence. Policy Research Working Paper Series 5015” World Bank, Washington, DC.

Georgiadis, G., Gräb, J., y Trottner, F. (2014): "Global Value Chain Participation and Current Account Imbalances", Working Paper.

Gereffi, G. (2014): "A Global Value Chain Perspective on Industrial Policy and Development in Emerging Markets", Duke Journal of Comparative and International Law, v. 24, p. 433.

Gereffi, Gary, John Humphrey, Raphael Kaplinsky, and Tim Sturgeon (2001): "Introduction: Globalisation, Value Chains and Development," IDS Bulletin, 32(3): 1-8.

Gereffi G., Humphrey J. y Sturgeon T. (2005): "The Governance of Global Value Chains”, Review of International Political Economy, vol. 12, no 1, pp. 78104.

Goldstein, M. y Khan, M. (1985): "Income and Price Effects in Foreign Trade", Handbook of International Economics, vol II, pp 1041-105.

Haltmaier, J. (2015): "Have Global Value Chains Contributed to Global Imbalances?”, International Finance Discussion Papers 1154, http://dx.doi. org/10.17016/IFDP.2015.1154.

Hausman R., Pritchett L y Rodrik D. (2005): "Growth Accelerations”, Journal of Economic Growth, 10, pp. 303-329.

Hernández R., Martinez J. y Mulder N. (2014): Global Value Chains and World Trade: Prospects and Challenges for Latin America, Santiago de Chile: ECLAC Books, No. 127 (LC/G.2617-P).

Hopkins, T. y Wallerstein, I. (1977): "Patterns of Development of the Modern World-System", Review (Fernand Braudel Center), vol. 1, no 2, pp. 111 145. 
Humphrey J. y Schmitz H. (2002): "How Does Insertion in Global Value Chains Affect Upgrading in Industrial Clusters?” Regional Studies, vol. 36, no 9, pp. 1017-1027.

Irwin, D. (2002): "Long-run Trends in World Trade and Income", World Trade Review, 1(1), pp. 89-100.

Kharroubi, E. (2011): "The Trade Balance and the Real Exchange Rate", BIS Quarterly Review, September.

Koopman, R.; Powers, W.; Wang, Z. y Wei, S. (2010): "Give Credit to Where Credit is Due: Tracing Value Added in Global Production Chains", NBER Working Paper No. 16426.

Lopez-Villavicencio, A. y Mignon, V. (2018). "Do Global Value Chains Amplify Global Imbalances?", EconomiX Working Papers 2018-38, University of Paris Nanterre, EconomiX.

McCombie, J. (1993): "Economic Growth, Trade Intelinkages, and the Balanceof-Payments Constraint", Journal of Post Keynesian Economics, vol.15, núm. 4, Nueva York, Sharpe, pp. 471-504.

OECD (2013): Wiod Database on Trade in Value-added, Londres, diponible en: http://goo.gl/2EGBRg.

Prebisch, R. (1968): El desarrollo económico de la América Latina y algunos de sus principales problemas, Fondo de Cultura Económica, México, (1949).

Smichowski, B. C., C. Durand y S. Knauss (2016): "Uneven Development Patterns in Global Value Chains: An Empirical Inquiry Based on a Conceptualization of GVCs as a Specifc form of the Division of Labor", en: 28th Annual Conference of the Society for the Advancement of Socio-Economics (SASE), Berkeley, 24-26 de junio.

Thirlwall, A. (1979): "The Balance of Payments Constrained Growth as an Explanation of International Growth Rate Differences", Banca Nazionale del Lavoro Quarterly Review 128, pp. 48-53.

Wallerstein I. (1974): "The Rise and Future Demise of theWorld Capitalist System: Concepts for Comparative Analysis", Comparative Studies in Society and History, vol. 16, no 2, pp. 387-415. 


\section{ANEXO}

TABLA A1: Países INCLUIDOS EN EL ESTUdio

\begin{tabular}{|l|l|l|}
\hline \multicolumn{3}{|c|}{ Ingresos Altos } \\
\hline Austria & Hungrīa & Nueva Zealanda \\
\hline Australia & Islandia & Noruega \\
\hline Bélgica & Irlanda & Polonia \\
\hline Canadā & Italia & Portugal \\
\hline Croacia & Japón & España \\
\hline Dinamarca & Letonia & Suecia \\
\hline Estonia & Lituania & Suiza \\
\hline Finlandia & Luxemburgo & Reino Unido \\
\hline Francia & Malta & Estados Unidos \\
\hline Alemania & Holanda & \\
\hline
\end{tabular}

\begin{tabular}{|l|l|}
\hline \multicolumn{2}{|c|}{ Ingresos Medios } \\
\hline Brasil & India \\
\hline Bulgaria & Filipinas \\
\hline Colombia & Argentina \\
\hline Costa Rica & Chile \\
\hline Malasia & Grecia \\
\hline Mēxico & Israel \\
\hline Perū & Arabia Saudita \\
\hline Rumania & Eslovaquia \\
\hline Rusia & Singapur \\
\hline Turquía & \\
\hline
\end{tabular}

Tabla A2: Resultados del teSt de Hausmann para efECtos fiJos/aleatorios.

\begin{tabular}{lcccc}
\hline \multirow{2}{*}{ VARIABLES } & \multicolumn{5}{c}{ Balanza Comercial } \\
\cline { 2 - 5 } & fe & (B) & $\begin{array}{c}(\mathrm{b}-\mathrm{B}) \\
\text { Difference }\end{array}$ & $\begin{array}{c}\text { sqrt(diag(V_b-V_B) } \\
\text { S.E. }\end{array}$ \\
\hline$Y^{*}$ & 1.07 & 2.091 & -1.02 & 1.24 \\
$Y$ & -1.054 & -2.963 & 1.91 & 0.49 \\
TCRM & -0.733 & -0.328 & -0.41 & 0.21 \\
CGV & -0.207 & -1.103 & 0.90 & 1.22 \\
& & & & \\
\hline Observations & 816 & 816 & & \\
Number of id & 48 & 48 & & \\
\hline
\end{tabular}

$\mathrm{b}=$ consistent under $\mathrm{Ho}$ and $\mathrm{Ha}$;

$\mathrm{B}=$ inconsistent under $\mathrm{Ha}$, efficient under $\mathrm{Ho}$;

Test: Ho: difference in coefficients not systematic

$$
\begin{aligned}
\operatorname{chi} 2(4) & =(b-B)^{\prime}\left[\left(V_{-} b-V_{-} \_B\right)^{\wedge}(-1)\right](b-B) \\
& =19.69
\end{aligned}
$$

Prob $>$ chi $2=0.0006$ 
TABLA A3: EstimaCiOnES UTILIZANDO LA VARIABLE ICE

\begin{tabular}{|c|c|c|c|c|c|c|}
\hline & \multicolumn{6}{|c|}{ Balanza Comercial } \\
\hline & (i) & (ii) & (iii) & (iv) & (v) & (vi) \\
\hline \multirow[t]{2}{*}{$\mathrm{Y}^{*}$} & 1.089 & $1.057^{*}$ & $1.093^{*}$ & 1.055 & $0.951 *$ & $0.973^{*}$ \\
\hline & (0.658) & (0.533) & $(0.265)$ & $(0.317)$ & $(0.254)$ & $(0.271)$ \\
\hline \multirow[t]{2}{*}{ Y } & $-1.171 * *$ & $-1.069 * *$ & $-1.081 * *$ & $-1.005^{* *}$ & $-0.993 * *$ & $-1.017^{* *}$ \\
\hline & $(0.414)$ & $(0.058)$ & $(0.072)$ & $(0.026)$ & $(0.061)$ & $(0.050)$ \\
\hline \multirow[t]{2}{*}{ TCRM } & $-0.874^{*}$ & $-0.811^{*}$ & $-0.725^{* *}$ & -0.952 & $-0.973^{*}$ & $-0.901^{* *}$ \\
\hline & $(0.125)$ & $(0.741)$ & $(0.032)$ & $(0.083)$ & $(0.068)$ & $(0.114)$ \\
\hline \multirow[t]{2}{*}{ ICE } & $-0.104^{*}$ & $-0.119^{*}$ & $-0.108 *$ & $-0.106^{*}$ & $-0.099 * *$ & $-0.086^{*}$ \\
\hline & $(0.077)$ & $(0.124)$ & $(0.081)$ & $(0.049)$ & $(0.043)$ & $(0.091)$ \\
\hline \multirow[t]{2}{*}{ ICEXTCRM } & & & & -0.056 & $-0.073^{*}$ & $-0.081^{*}$ \\
\hline & & & & $(0.245)$ & $(0.136)$ & $(0.178)$ \\
\hline \multirow[t]{2}{*}{ Constant } & 0.176 & $1.513^{*}$ & 0.181 & $0.044^{*}$ & $0.255^{*}$ & $0.158^{*}$ \\
\hline & (0.158) & $(0.282)$ & $(1.209)$ & $(0.474)$ & $(0.134)$ & (1.993) \\
\hline Time fixed effects & No & Yes & Yes & No & Yes & Yes \\
\hline Country fixed effects & No & No & Yes & No & No & Yes \\
\hline Observations & 816 & 816 & 816 & 816 & 816 & 816 \\
\hline Number of id & & 48 & 48 & & 48 & 48 \\
\hline
\end{tabular}

Nota: Errores estāndares robustos entre paréntesis. ${ }^{*} \mathrm{y} * *$ denotan significatividad estadística al $5 \%$ y $1 \%$, respectivamente. 
TABLA A4: ESTIMACIONES SEGŨN NIVEL DE INGRESO

\begin{tabular}{|c|c|c|c|c|c|c|c|c|}
\hline & \multicolumn{4}{|c|}{ Ingreso Alto } & \multicolumn{4}{|c|}{ Ingreso Medio } \\
\hline & (i) & (ii) & (iii) & (iv) & (v) & (vi) & (vii) & (viii) \\
\hline \multirow[t]{2}{*}{$Y^{*}$} & 0.881 & $0.842^{*}$ & 1.034 & $1.092^{*}$ & $0.991 *$ & $1.017^{*}$ & $1.204^{*}$ & $1.110^{*}$ \\
\hline & $(0.292)$ & $(0.312)$ & $(0.182)$ & $(0.235)$ & $(0.205)$ & $(0.194)$ & $(0.258)$ & $(0.255)$ \\
\hline \multirow[t]{2}{*}{ Y } & $-0.731^{* *}$ & $-0.779^{*}$ & $-0.824^{*}$ & $-0.899 *$ & $-1.089 * *$ & $-1.102 * *$ & $-1.133^{* *}$ & $-1.225^{* *}$ \\
\hline & $(0.074)$ & $(0.109)$ & $(0.087)$ & $(0.099)$ & $(0.150)$ & $(0.109)$ & $(0.114)$ & $(0.105)$ \\
\hline \multirow[t]{2}{*}{ TCRM } & $-1.017^{*}$ & -0.998 & $-0.971^{*}$ & -0.984 & $-0.799 * *$ & $-0.804^{* *}$ & $-0.894^{*}$ & $-0.983^{*}$ \\
\hline & $(0.071)$ & $(0.108)$ & $(0.107)$ & $(0.104)$ & $(0.491)$ & $(0.232)$ & $(0.124)$ & (0.115) \\
\hline \multirow[t]{2}{*}{ CGV } & $-0.092 * *$ & $-0.089^{*}$ & $-0.078^{*}$ & $-0.085^{*}$ & $-0.124^{*}$ & $-0.115^{*}$ & $-0.109 *$ & $-0.121 *$ \\
\hline & $(0.049)$ & $(0.082)$ & $(0.158)$ & $(0.299)$ & $(0.122)$ & $(0.171)$ & $(0.224)$ & $(0.412)$ \\
\hline \multirow[t]{2}{*}{ CGVxTCRM } & $0.123^{*}$ & $0.091^{* *}$ & & & $0.105^{*}$ & $0.093^{*}$ & & \\
\hline & $(0.134)$ & $(0.115)$ & & & $(0.324)$ & $(0.145)$ & & \\
\hline \multirow[t]{2}{*}{ CGVxY } & & & $0.041^{*}$ & $0.055^{*}$ & & & $0.052^{*}$ & $0.083^{*}$ \\
\hline & & & (0.029) & $(0.074)$ & & & (0.149) & $(0.211)$ \\
\hline \multirow[t]{2}{*}{$\mathrm{CGVxY}^{*}$} & & & $-0.059^{*}$ & $-0.063^{*}$ & & & $-0.072^{*}$ & $-0.078^{*}$ \\
\hline & & & (0.109) & (0.113) & & & $(0.147)$ & (0.193) \\
\hline
\end{tabular}

Constant

\begin{tabular}{|c|c|c|c|c|c|c|c|c|}
\hline $\begin{array}{l}\text { Time fixed } \\
\text { effects }\end{array}$ & Yes & Yes & Yes & Yes & Yes & Yes & Yes & Yes \\
\hline $\begin{array}{l}\text { Country } \\
\text { fixed effects }\end{array}$ & No & Yes & No & Yes & No & Yes & No & Yes \\
\hline Observations & 493 & 493 & 493 & 493 & 323 & 323 & 323 & 323 \\
\hline Number of id & 29 & 29 & 29 & 29 & 19 & 19 & 19 & 19 \\
\hline \multicolumn{9}{|c|}{$\begin{array}{l}\text { Nota: Errores estándares robustos entre paréntesis. }{ }^{*} \text { y }{ }^{* *} \text { denotan significatividad estadística al } \\
5 \% \text { y } 1 \% \text {, respectivamente. }\end{array}$} \\
\hline \multicolumn{9}{|c|}{$\begin{array}{l}\text { La clasificaciōn de los paîses por niveles de ingreso se basa en la realizada por el Banco Mundial. En } \\
\text { ingreso medio se incluyen aquellos clasificados en las categorías de "Ingreso Medio-Alto" e "Ingreso } \\
\text { Medio-Bajo" por el Banco Mundial. Finalmente, los países quedan agrupados como se especifica } \\
\text { en la tabla A1. }\end{array}$} \\
\hline
\end{tabular}

